\title{
Non-invasive tests and markers for liver fibrosis
}

\author{
Nagwa S. Ahmed, Mohamed Ahmed Abdelmoaty, Tahia H. Saleem, \\ Ghada M. Galal and Amira Morad
}

\begin{abstract}
Chronic liver disease is characterized by liver fibrosis, which may lead to cirrhosis. Conventional serum-based liver function tests do not give information on either the presence or the rate of progress of liver fibrosis. The reference diagnostic test to detect fibrosis is liver biopsy, a procedure subject to various limitations, including risk of patient injury and sampling error.

Serum markers have been evaluated for the determination of fibrosis either singly or combined as a panel of markers, however diagnostic accuracy is greatest in studies using a panel together with an algorithm, which generates a predictive score. Serum marker models, especially those targeted at hepatitis $\mathrm{C}$, have multiplied in spectacular fashion over the last five years, with most models regularly achieving a median area under the receiver operating characteristic curve (ROCC) of $\mathbf{0 . 8 0}$ versus liver biopsy. Five years after publication of the first major serum marker model, the first study to document clinical outcomes reported that applying the model to hepatitis $\mathrm{C}$ patients improved prediction of decompensated cirrhosis and survival compared to liver biopsy.

An obstacle to widespread adoption of serum marker models has been the lack of uniform performance indicators, such as diagnostic odds ratios and likelihood ratios. This review highlights the most well-established noninvasive biomarkers to-date, with a particular emphasis on serum
\end{abstract}

\section{Introduction}

Chronic liver diseases of differing etiologies are among the leading causes of morbidity and mortality worldwide [1-5]. Chronic liver disease progresses through different pathological stages that vary from mild hepatic inflammation without fibrosis to advanced hepatic fibrosis and cirrhosis [6-8]. Assessment of the stage of liver disease is important for diagnosis, treatment, and follow-up both during treatment and after cessation of treatment. A liver biopsy is the oldest and most accurate method used to evaluate liver histology and the progression of chronic liver disease. Furthermore, different histological scoring systems have been developed and modified [9-12].

Liver biopsy - an "imperfect" gold standard

Biopsy has been the gold standard in diagnosing and staging liver fibrosis and also has the advantage of assessing other disease aspects as well including inflammation, steatosis and necrosis. However, biopsy is often considered an "imperfect" gold standard since it suffers from intra/inter-observer variability and is associated with several complications including hospitalization in $\mathbf{1 - 5 \%}$ of cases and mortality in $\mathbf{0 . 0 1 - 0 . 1 \%}$ of cases.[13-14] In addition, biopsy is subject to sampling error as the 1-2 pieces of $1 \mathrm{~cm}$ long tissue only accounts for 1/50,000 of the liver volume.[15] For example, laparoscopic liver biopsies from the right and left lobes of $\mathrm{HCV}$ patients differed by at least one Metavir stage in $33 \%$ of cases,[16] and higher sampling variability has been reported for NASH and biliary fibrosis.[1718] Sampling error even exists for 
advanced stages of disease as biopsy can lead to under-staging of cirrhosis in up to $20 \%$ of patients.[19] Importantly, repeated biopsies to evaluate disease progression or response to treatment are impractical due to the increased risk of complications and poor patient compliance. For all of these reasons, noninvasive strategies that can repeatedly assess liver fibrosis throughout the entire organ are urgently needed to assess disease stage, monitor treatment response, and determine prognosis.

Recently many noninvasive markers (NIMs) for assessing liver fibrosis have been developed, and they are frequently used in clinical practice. They have been validated in different studies, and some were found to be highly accurate in the assessment of liver fibrosis compared with liver biopsies [20-23], which have always been used as the standard reference method for evaluating the accuracy of noninvasive methods. Most non-invasive markers of liver fibrosis were developed with the aim of discriminating between 'insignificant", (F0-F1)by METAVIR and clinically "significant" fibrosis (> F2)by
METAVIR or of identifying or excluding established cirrhosis in patients with well compensated chronic liver disease [24].

On the other hand, patients with F0-F1 fibrosis usually do not progress or progress much slowly and are often not as aggressively offered treatment [2528]. However, as therapy for $\mathrm{HCV}$ improves, the clinical need for a biopsy may be less apparent.For example, genotype $\mathbf{2}$ and $\mathbf{3}$ patients have a greater than $70 \%$ sustained virologic response with pegylatedinterferons and ribavirin, and in uncomplicated cases a rationale can be made to treat all patients without liver biopsy and only to biopsy those who fail treatment [23\&29]. As newer, better tolerated, and more efficacious therapies are being developed, the need for doing biopsy to all $\mathrm{HCV}$ patients to grade and stage the disease may become redundant.

- Characteristics of an ideal marker of liver fibrosis

The great majority of studies have investigated the diagnostic value of serum markers of liver fibrosis. The requirements for an ideal marker are shown in table 1.[30]

- Liver specific

- Noninvasive

- Easy to perform

- Measurable by sensitive, reproducible and fast methodology

- Serum levels are independent of alterations in liver, renal or reticuloendothelial function

1. Capacity to reflect one or more of the following processes:

- stage of fibrosis;

- activity of matrix deposition;

- activity of matrix removal.

2. Possibility to follow the progression or regression of fibrosis in natural evolution or under treatment

The accuracy of a test is given as the area under the curve (AUC)of the receiver operator characteristic (ROC).An ideal marker would have an AUC of $\mathbf{1 . 0}$ and thus a $\mathbf{1 0 0} \%$ 
sensitivity and specificity.The great majority of proposed biochemical markers have an AUC between 0.80-0.85 and have value not for staging the disease, but rather for differentiating insignificant (F0/F1)from significant fibrosis (F2-F4 Metavir).Moreover, the indeterminate results occur mainly in patients who have F1 to F2 disease.Taking into account that the value of a biomarker is validated against the biopsy, which has an accuracy of about $\mathbf{8 0 \%}$. It is improbable that a biomarker has a better performance than liver biopsy for staging fibrosis [25]

According to Afdhal and Nune, 2004 none of the available markers fulfill these ideal criteria.None are liver specific, so there may be significant contribution from non-hepatic sources, including bone, joints, skin, and lungs.Levels of these markers are altered by changes in their clearance, metabolism, and excretion.For instance, both the liver (80\%) and the kidneys (20\%)clear hyaluronan and the removal from circulation is also dependent upon binding to specific receptors by hepatic endothelial cells [26].Increased hyaluronan levels occur in the post prandial state, presumably as a result of competition for these receptors[27].

Their relationship to the total matrix content of the liver and to the activity of fibrogenesis or degradation is usually mixed.Indeed, in the absence of a golden standard of matrix turnover, it is not possible to assess the relationship of the levels of these markers to ongoing matrix remodeling and new matrix deposition or removal.Thus from a practical and research standpoint, it would be ideal to have marker(s)that can: (a) Noninvasively stage the degree of liver fibrosis; or (b) Reflect the rate of matrix deposition or removal, both to monitor the impact of therapies and to give prognostic information.It is unlikely that any single marker can fulfill both of these ideal criteria [28]

Serum markers:

Serum markers of liver fibrosis may be divided in two categories:

- $\quad$ Direct markers, which reflect ECM turnover and

- $\quad$ Indirect markers, which reflect alterations in hepatic function, but not directly

ECM metabolism [25]

I- Direct serological markers of liver fibrosis

These markers are supposed to be directly involved in the deposition and removal of ECM, i.e.in fibrogenesis and fibrolysis.They include markers of matrix metabolism as well as cytokines.Fibrosis markers can be classified according to their molecular structure (Table 2). 
Table 2. Fibrosis markers [29]

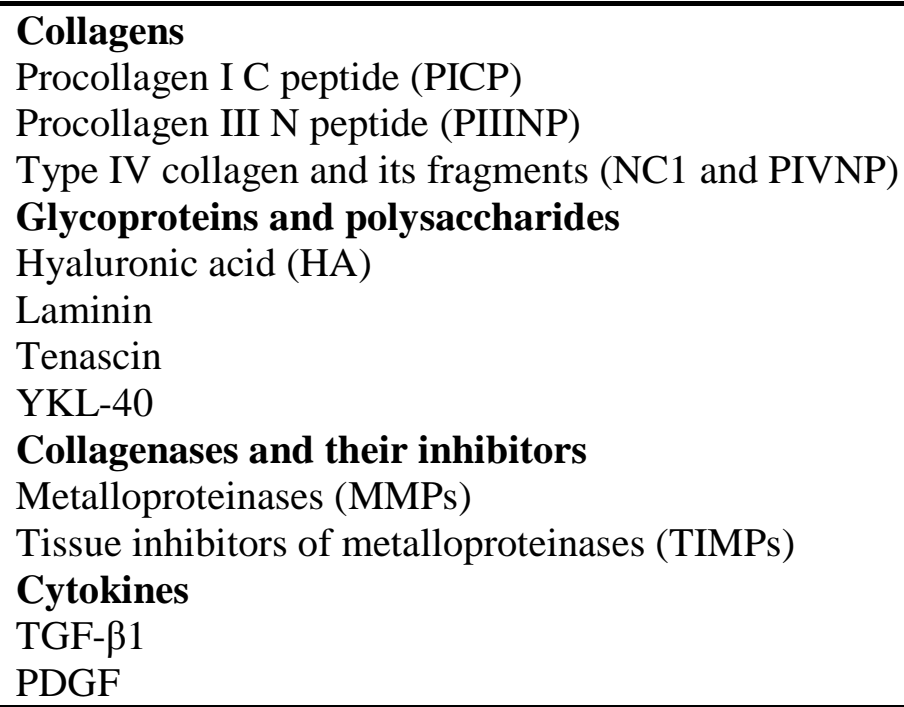

It is very difficult to make a clear delimitation between markers of ECM deposition and degradation.Serum levels of direct markers reflect simultaneously both processes as well as the total mass of ECM undergoing remodeling. The assessment of direct markers could be useful for staging liver disease and for assessing the effect of treatment and predicting disease progression [25]

A. Markers associated with matrix deposition

Several studies have investigated the value of procollagen peptides.During synthesis of collagen, procollagen suffers an enzymatic cleavage at both the carboxy and aminoterminal ends by two different enzymes: procollagen $-\mathrm{C}-$ proteinase and procollagen- $\mathrm{N}$ proteinase.The peptides released into the serum :procollagen type I carboxy terminal peptide and procollagen type III amino-terminal peptide can be used as a measure of matrix deposition [29]

\section{1) Procollagen type I carboxy} terminal peptide (PICP)

PICP has little value in the diagnosis of chronic hepatitis and is elevated in cirrhosis, quantifying disease severity or indicating the alcoholic etiology [30]

2) Procollagen type III aminoterminal peptide (PIIINP)

Serum levels of PIIINP were extensively studied alone or in combination with different other markers and the results showed the correlation between their levels and histological stage of hepatic fibrosis in alcoholic liver disease, viral hepatitis and primary biliary cirrhosis [29].

3) Serum type IV collagen

Type IV collagen is an important component of ECM.Unlike type I and III collagens, which are processed by proteolysis, type IV collagen is deposited intact in the matrix and the serum component of type IV collagen reflects matrix degradation [30]. Irrespective of the methods used for determination, serum levels of type IV collagen had a positive correlation with the degree of hepatic fibrosis in patients with chronic viral hepatitis [25].

\section{4) Laminin}

A major non-collagenous glycoprotein synthetized by HSC, laminin is 
deposited in the basement membrane of the liver and increases during fibrosis around the vessels, in the perisinusoidal spaces and the portal tract.Serum laminin levels and pepsin-resistant fragment of laminin (laminin P1)are elevated in chronic liver diseases irrespective of etiology: viral or alcoholic and reflect an increase in perisinusoidal fibrosis.Serum levels of laminin correlate with the severity of fibrosis and liver inflammation in chronic hepatitis $\mathrm{C}$, and are superior to serum ALT in reflecting liver injury [31].

\section{5) Hyaluronic acid}

Hyaluronic acid (HA)is a glycosaminoglycan, component of the ECM, synthetized by HSC.In normal circumstances the endothelial cells of the liver sinusoid are the site of HA uptake and degradation [32]. Increased levels of HA are due to decreased hepatic removal, increased production or both.High levels of serum HA were detected in patients with liver diseases of different etiologies and particularly in those with cirrhosis [33].Serum levels of HA were shown to be related not only to the stage of fibrosis but also to the degree of necroinflammation[30].

\section{6) YKL-40 (chondrex)}

YKL-40 is a mammalian member of a chitinase family

(18glycosylhydrolases).YKL-40 is produced in a wide variety of cell types and especially in cells located in tissues with increased remodelling/ degradation or inflammation of the ECM.The cellular source in the liver is supposed to be activated HSC.Its physiological function is unknown, but YKL-40 may contribute to tissue remodelling, acts as a growth factor for fibroblasts, acts synergistically with insulin-like growth factor, as a chemoatractant for endothelial cells and has a role in angiogenesis.In liver diseases, serum levels of YKL-40 were closely related to the degree of fibrosis histologically documented, the highest values being found in severe fibrosis [34].

In chronic $\mathrm{HCV}$ infection, serum levels greater than $\mathbf{2 8 4 . 8} \mathrm{ng} / \mathrm{ml}$ predict cirrhosis with a sensitivity of $80 \%$ and specificity of $\mathbf{7 1} \%$ and have a negative predictive value (NPV)of $\mathbf{7 8 \%}$.Unlike PIIINP and HA, serum YKL-40 is significantly elevated in the subset of alcoholic cirrhotic patients who have also alcoholic hepatitis and is the best of these serological markers in discriminating between patients with mild fibrosis and those with no fibrosis [34\&35]

B- Markers associated with matrix degradation

Products of matrix degradation result from the activity of a family of enzymes:matrix metalloproteinases (MMPs).Synthesized intracellularly and secreted as pro-enzymes, MMPs are activated by a proteolytic cleavage by membranetype matrix metaloproteinase 1 (MT1-MMP)or plasmin and inhibited by binding to specific tissue inhibitors of metalloproteinase (TIMPs).Considering their substrate specificity there are five categories of MMPs [35]:

3. Interstitial collagenases (MMP-1, -8, -13),

4. Gellatinases(MMP-2, $\quad-9$ and fibroblast activation protein).

5. Stromelysins (MMP-3, -7, -10, -11)

6. Membrane type (MMP-14, -15, 16, -17, -24, -25)

7. Metalloelastase (MMP -12)

The MMPs and their inhibitors are involved in the control of matrix degradation [36]TIMPs can irreversibly bind the proenzyme or active forms of MMPs and inactivate them.Excess 
production of TIMPs relative to MMPs may be an important factor for progression of liver fibrosis [37]HSC are the principal source of MMP-2 in the human liver and activation of MMP-2 require interaction with hepatocytes.TIMP-1 is produced by HSC and hepatocytes [36and 37]Regarding the diagnostic value of MMP-2 and TIMP-1, one study reported that MMP-2 levels were elevated only when cirrhosis had developed, while TIMP-1 had a diagnostic value in detecting earlier stage of fibrosis.Also, this study revealed that TIMP-1 levels had a strong correlation with histological inflammatory scores and that MMP-2 level had no relationship to the stage of fibrosis in the non-cirrhotic liver [37] Older studies which investigated the role of TIMP-1 in patients with various liver disease, comparing TIMP-1 with PIIINP, type IV collagen, laminin P1 and the histological aspect, suggested that the serum levels of TIMP1 may be useful to estimate hepatic fibrogenesis associated with active inflammatory activity. Also, the serum levels of TIMP-1 were shown to correlate positively with the degree of fibrosis and a striking increase in serum TIMP-1 levels was observed in the late stage of fibrosis, but not in the mild stage the ratio of TMP-1/MMP-1 could be useful in the diagnosis of hepatic fibrosis [38].

C- Cytokines and chemokines associated with hepatic fibrosis

1- TGF- $\beta 1$

Transforming growth factor- $\beta \mathbf{1}$ (TGF$\beta 1)$ is a homodimetric polypeptide that is secreted in an inactive form which requires activation. It has pleiotropic effects through membrane receptors on a wide variety of cells .In hepatic pathology, TGF- $\beta 1$ is the most important stimulus for the production of ECM by
HSC and it is also an inhibitor of hepatocyte growth and proliferation [39].In the liver biopsy from patients with chronic liver disease, TGF- $\beta \mathbf{1}$, mRNA levels correlate with type I collagen mRNA (40]. The value of serum TGF-b1 levels has some limitations related to the contamination of the sample by TGF $-\beta$ from platelets, the interference with plasmin activity in the plasma that increases the amount of TGF- $\beta 1$ through opening LAPTGF $-\beta$ complex,

the binding of TGF- $\beta$ at the sites of injury to ECM and to vascular endothelium, the sequestration by soluble proteins and the complicated clearance of TGF- $\beta 1$.These factors explain why plasma levels of TGF $\beta \mathbf{1}$ are unlikely to be of diagnostic value [40]However, some authors showed a good correlation between serum levels of total TGF $-\beta$, and Knodell scores and also a correlation with the rate of fibrosis progression[41].Moreover, other authors established cut-off values with prognostic significance for patients with no progression of fibrosis and those with progressive disease.A TGF- $\beta 1$ level below $75 \mathrm{ng} / \mathrm{ml}$ was predictive for stable disease [42].

\section{2- PDGF}

Platelet derived growth factor (PDGF)is the main stimulus of HSC proliferation and migration and is upregulated following liver injury. PDGF-BB is the main subunit with the most important role for the signalling pathway in HSC [43].The serum level of PDGF-BB was found to have the best value for assessment of hepatic fibrosis, when compared to other eight markers [38]

3- Intracellular adhesion molecule-1 (ICAM-1)andvascular adhesion molecule-1 (VCAM-1) :-

Few studies have evaluated the 
relationship between the levels of soluble adhesion molecules and liver inflammation and fibrosis [44]. Increased expression of intracellular adhesion molecule-1 (ICAM-1)occurs in virus infected hepatocytes; whereas expression of vascular adhesion molecule-1 (VCAM-1)may be seen in association with progressive hepatic fibrosis .It is thought that increased VCAM-1 expression occurs in association with capillarization of the sinusoidal spaces and fibrous septa [44] the correlation of individual direct markers with fibrosis and inflammation is shown in (Table 14).

Table 3. The correlation of direct serum markers with the histological substrate (25)

\begin{tabular}{|l|l|l|l|}
\hline Markers & Disease & Fibrosis & Inflammation \\
\hline Procollagen type I & CVH* & + & + \\
\hline PIIINP & CVH & +++ & +++ \\
\hline Type I collagen & Variousliverdisease & + & + \\
\hline TypeIV collagen & CVM & ++ & ++ \\
\hline Laminin & CVM & +++ & ++ \\
\hline Hyaluronic acid & CVM & +++ & + \\
\hline YKL-40 & CVH & +++ & + \\
\hline
\end{tabular}

$* \mathrm{CVH}=$ chronic viral hepatitis. $\mathrm{CVM}=$ cardiovascular mortality

\section{D-Other serum proteins:}

\section{1- $\alpha$-2-Macroglobulin ( $\alpha-2-\mathrm{M})$}

This is a high molecular weight protein synthesized in hepatocytes and stellate cells which is reasonably abundant in human serum, where normal levels are typically from $\mathbf{0 . 6 6}$ to $2.65 \mathrm{~g} / \mathrm{L}$. The functions of $\alpha-2-\mathrm{M}$ are not well understood but it does inhibit the catabolism of matrix proteins by acting as a broadspectrum inhibitor of nearly all enzymes that split proteins internally (endoproteases).Serum levels increase with the degree of liver fibrosis [45]

\section{2- Apolipoprotein A1 (Apo A1)}

This is the major protein component found in high-density lipoprotein.Serum concentrations are negatively associated with liver fibrosis, i.e.levels decrease as the extent of fibrosis increases.Decreased levels are also seen in uncontrolled diabetes, nephrotic syndrome, some diets and smoking [49].

\section{3- Haptoglobin (Hap)}

This serum protein binds any free haemoglobin present in the circulation.Hap is an acute phase protein whose concentrations increase in a wide variety of inflammatory conditions and in nephrotic syndrome. Concentrations decrease in invivohaemolysis whether caused by autoimmune, iso-immune or mechanical reasons.Hap levels also decrease with increasing stages of fibrosis [47].

Limitations of the serum direct markers of liver fibrosis

Using either a single marker or a combination of tests, direct markers 
have some limitations [48]

1. They reflect the rate of matrix turnover, not only deposition, and have the tendency to be more elevated when there is an associated high inflammatory activity.As a consequence, extensive matrix deposition might not be detected in the presence of minimal inflammation.

2. They are not liver-specific and their serum levels may be elevated in the presence of concomitant sites of inflammation.

3. Serum levels of markers depend on clearance rates, which are influenced by dysfunction of endothelial cells, impaired biliary excretion or renal function.

4. One of the main limitations to the clinical use of direct markers of liver fibrosis is that they are not routinely available in all hospital settings [25].

II- Indirect markers of liver fibrosis

According to [48]liver fibrosis may be predicted by using a single routine laboratory test that reflects alteration in hepatic function, or a combination of such tests .These markers include-:

1- Serum ALT levels

Although serum ALT levels generally reflect liver injury, the correlation between ALT levels and necroinflammatory and fibrosis score is poor, especially in chronic viral $\mathrm{C}$ infection [49]. However, an extensive study established that ALT levels had a good sensitivity and specificity for the prediction of histologic score.ROC analysis showed that the best theoretical ALT threshold with the best histologic predictive value is 2.25 times the upper limit of normal, but it implies the overlooking of $\mathbf{2 8} \%$ of patients with a histologic score greater than A1F1 Metavir.At the same time, among patients with persistently normal ALT levels, about $26 \%$ have a histologic score greater than $\mathrm{A} 1 \mathrm{~F} 1$, and a liver biopsy must be taken into consideration [50].The relative increase in AST is probably related to both reduced clearance of AST by hepatic sinusoidal cells as well as to mitochondrial dysfunction [51].

\section{2- AST /ALT ratio}

Assay of AST levels had a stronger correlation than ALT with hepatic fibrosis [52].The increase in ALT levels isrelated to mitochondrial dysfunction and to reduced clearance of AST by hepatic sinusoidal cells.Reversal of the AST /ALT ratio was reported in patients who progress from chronic hepatitis to liver cirrhosis and the AST/ALT ratio of more than $\mathbf{1}$ had a good predictive value for advanced fibrosis or cirrhosis[53].

\section{3- Platelet count (PLT)}

Thrombocytopenia is a valuable marker of advanced liver disease, but it may be related to many mechanisms as hypersplenism, myelosuppression by $\mathrm{HCV}$, decreased thrombopoetin production, autoimmune process [53]Combined assessment of the AST/ALT ratio and PLT had a high diagnostic value for cirrhosis [54].

\section{4- Prothrombin index}

Prothrombin time as an index that reflects the synthesis capacity of the liver is one of the earliest indicators of liver cirrhosis .In the HALT-C study, a multivariate logistic regression model that comprised prothrombin time, PLT, AST/ALT ratio and alkaline phosphatase was predictive of cirrhosis. Prolonged prothrombin time correlated with the presence and size of esophageal 
varices.Prothrombin time is also a part of different composite indices [54].

\section{5- PGA Index}

PGA index was the original index of hepatic fibrosis described in 1991.It was devised as a simple biological index for detecting alcoholic liver disease in drinkers[46]. It combines the measurement of the prothrombin index, gamma glutamyltransferase $(\gamma \mathrm{GT})$ and apolipoprotein A1 (PGA).It has been validated in patients with various chronic liver diseases, in particular alcohol (hence the use of $\gamma$ GT) [55].The diagnostic accuracy of the PGA score for detecting cirrhosis is reported between $66 \%$ and $72 \%$. This was subsequently modified to the PGAA index by the addition of $\alpha \mathbf{2}$-macroglobulin, which resulted in some improvement in its performance [45].Ofimportance, it was noted that the aetiology of the liver disease affected the performanceof some of these tests.The PGA and PGAA scores performed better in alcoholic than viralliver disease, whereas, the prothrombin index and hyaluronan levels performed similarlyin both disease groups. The AST/ALT ratio performed better in viral liver diseases than in alcoholic liver diseases [25].

\section{6- Fibrotest}

Fibrotest, is the most widely known, and the most validated of the tests of the noninvasive evaluation of liver fibrosis.In the study, a total of $\mathbf{1 1}$ markers were assessed: $\alpha_{2}$ macroglobulin, AST, ALT, $\gamma$ glutamyltransferase, total bilirubin, albumin, $\alpha_{1}$ globulin, $\alpha_{2}$ globulin, $\beta$ globulin, $\quad \gamma$ globulin, and apolipoprotein A1 .The $\alpha_{2}$ globulins mainly consisted of $\alpha_{2}$ macroglobulin and haptoglobin. The most informative markers were, in the decreasing rank: $\alpha_{2}$ macroglobulin, haptoglobin, GGT, $\gamma$ globulin, total bilirubin, and apolipoprotein A1 [47].

By selecting the upper and lower cut off values, the authors were able to categorize patients into three groups[47]

(1)Those in which there was a high certainty of mild liver disease (METAVIR F0-1).

(2)Those with high certainty of significant fibrosis (METAVIR F2-4).

(3) Group of patients who could not be adequately characterized and in whom liver biopsy would be necessary, the indeterminate group.

With the best index, a high negative predictive value $(\mathbf{1 0 0} \%$ certainty of absence of F2, F3, or F4 fibrosis)was obtained in scores ranging from zero to $\mathbf{0 . 1 0}$, and high positive predictive value $(>\mathbf{9 0} \%$ certainty of presence of $\mathrm{F} 2, \mathrm{F3}$, or F4 fibrosis)for scores ranging from 0.60 to $\mathbf{1 . 0 0}$. The detection of significant fibrosis F2 or greater had a $\mathbf{7 5} \%$ sensitivity and $85 \%$ specificity. The assay performed somewhat better for the assessment of more advanced liver disease (METAVIR stages 3 and 4) [47].

\section{7- Acti Test}

Acti Test is a modification of the Fibrotest that incorporates ALT and reflects both necroinflammatory activity and liver fibrosis [47]. Both Acti Test and Fibrotest have shown reductions among those with sustained virological response to interferon and ribavirin therapies, supporting a role in following response to therapy [57]. Additionally, there was no difference between the area under the ROC's of Fibrotest/Acti Test according to the 
genotype or viral load.Thus the use of biochemical markers of liver fibrosis (Fibrotest), and necrosis (Acti Test)can be recommended as an alternative to liver biopsy for the assessment of liver injury in patients with chronic hepatitis $\mathbf{C}$ [58].

\section{8- Steato Test}

Developed by Poynard and colleagues, Steato Test incorporates the five components of Fibrotest $\quad(\alpha 2$ macroglobulin, haptoglobin, apolipoprotein $\quad \mathrm{A} 1, \quad \gamma$ glutamyltransferase, total bilirubin)and Acti Test (ALT)plus body mass index, serum cholesterol, triglycerides, and glucose adjusted for age and gender.Although the PPV of the Steato test is not that high, but still is significantly higher than those of other markers to evaluate steatosis, such as, GGT, ALT, and ultrasonography [58].

9- Forns index Forns et al.(2002) reported an index based on four readily available variables; age, platelet count, $\gamma$ glutamyltransferase, and cholesterol levels. The study was confined to patients with hepatitis $\mathrm{C}$ and included both test and validation cohorts. The authors constructed a simple score system applying a constant to the obtained formula:7.811 - $3.131 \times$ (platelet count $)+0.781 \times($ GGT $)+3.467 \times$ (age) $-\mathbf{0 . 0 1 4} \times($ cholesterol $[\mathrm{mg} / \mathrm{dL}])$

Half of the patients with chronic hepatitis $\mathrm{C}$ without significant liver fibrosis can be identified with high accuracy with this panel, and liver biopsy could have been avoided in more than one third of the patients [59]The performance of this index was compared to the Fibrotest the results of the Forns index were reproducible but performed slightly less well than Fibrotest.Major concerns about the Forns index include the impact of lipid abnormalities in patients with hepatitis $\mathrm{C}$, cholesterol altering medicines, and the reproducibility of platelet estimations [25].

10- APRI (AST to Platelet Ratio Index)

Wai et al. (2003)looked at a number of simple laboratory measurements and their relationship to two end points:significant liver fibrosis and cirrhosis. Based on their analysis of these laboratory tests, they found that the APRI was the simplest and the most accurate test for the evaluation of two end points. It is calculated as

follows:APRI = AST level / ULN x $\mathbf{1 0 0}$

\section{Platelet count $\left(\mathbf{1 0}^{\mathbf{9}} / \mathrm{l}\right)$}

Platelet counts and AST levels were found to be the most important predictors of significant fibrosis and cirrhosis.This novel index was developed to amplify the opposing effects of fibrosis on AST and platelet count.By using the optimized cut off values, significant fibrosis could be predicted accurately in $\mathbf{5 1} \%$ and cirrhosis in $81 \%$ of patients [60]

\section{1- Göteborg University} Cirrhosis Index (GUCI)

In this study, samples from 179 patients with chronic hepatitis $\mathbf{C}$ were analyzed using routinely available biochemical markers of liver disease, and compared with liver biopsy using the Ishak protocol. Multivariate logistic regression analysis revealed strong association between AST, platelet count and prothrombinINR.They developed the Göteborg University Cirrhosis Index (GUCI)according to the formula:( Normalized AST x prothrombin-INR x 100)/Platelet count (x 10\% $/ \mathrm{L})$ 
Using a cut-off value of $\mathbf{1 . 0}$, the sensitivity was $80 \%$, and the specificity $\mathbf{7 8} \%$ for the diagnosis of cirrhosis, and the negative predictive value and the positive predictive values were $97 \%$ and $31 \%$ respectively.The authors concluded that the GUCI score proved slightly superior for sensitivity, specificity, NPV, PPV, and the area under the receiver operating characteristic curve (ROC)for the prediction of cirrhosis and bridging fibrosis compared with AST to platelet ratio index (APRI)[61].

III- Panels with Indirect \& Direct markers of liver fibrosis:

Imbert-Bismut et al., 2001)suggested that the Combinations of direct and indirect markers enhance the accuracy of liver fibrosis detection

1- FibroSpect[62] in a retrospective cohort study evaluated the FibroSpect assay which involves three parameters: hyaluronic acid, TIMP1(tissue inhibitor of metalloproteinase 1), and $\alpha_{2}$-macroglobulin. These three markers were selected as having the best predictive accuracy for F2-F4 fibrosis (combined AUROC $=\mathbf{0 . 8 3 1}$ ). All patients were evaluable by the FibroSpect with no indeterminate values, and this is an advantage of the assay.Maximum sensitivity and specificity were seen at the two extreme spectrums of disease, stage 0 and stage 4.

It was concluded that the three marker panel may reliably differentiate chronic hepatitis $\mathrm{C}$ patients with moderate /severe fibrosis from those with no/mild fibrosis, although accurate delineation between stages was not possible [62]

FibroSpectII: In a reported retrospective study, hyaluronic acid,
YKL 40, and FibroSpect II comprising: hyaluronic acid, TIMP-1, and $\alpha_{2}$-macroglobulinwere assessed with Ishak stages and digital quantification of fibrosis (DQF).Among the serum markers, hyaluronic acid was effective in discriminating Ishak stages 0-1 and Ishak stages 2-3 compared with FSII, with area under the ROC curve of $\mathbf{0 . 7 6}$ versus $\mathbf{0 . 6 6}$ respectively.All three serum markers predicted advanced fibrosis and cirrhosis. YKL40 had the highest false positive rates in all categories of fibrosis [63]

2- European Liver Fibrosis Group assay: The ELF group reported a novel assay in an international multicenter cohort of 1021 patients with hepatitis $\mathrm{C}$, nonalcoholic fatty liver disease, and alcoholic liver disease.The authors aimed to develop a panel of sensitive automated immunoassays to detect matrix constituents and mediators of matrix remodeling in serum to evaluate their performance in the detection of liver fibrosis [64]. Discriminant analysis of a test set of samples was used to identify an algorithm combining age, hyaluronic acid, amino-terminal propeptide of type III collagen (PIIINP), and TIMP1.The authors concluded that assessment of liver fibrosis with multiple serum markers used in combination is sensitive, specific, and reproducible, suggesting that they may be used in conjunction with liver biopsy to assess a range of chronic liver diseases [64]

3- Fibrometer: In this landmark study, the authors studied patients with viral hepatitis, patients with alcoholic liver disease in the exploratory step, and the validating population patients with chronic liver disease due to 
HCV. The objective was to develop tests to characterize different fibrosis parameters (Fibrometer)in viral and alcoholic liver diseases. Measurements included $\mathbf{5 1}$ blood markers, and Fibrotest, Fibrospect, ELFG, APRI, and Forns scores.The clinically significant fibrosis was evaluated via Metavirstaging) F2-F4), and image analysis was used to determine the area of fibrosis (AOF)Significant correlation with METAVIR fibrosis stages (r=o.71) and Weaker correlations with histological activity $(\mathbf{r}=\mathbf{0 . 4 7})$ [65].

4- Hepascore: This model consists of bilirubin, $\gamma$ glutamyltransferase, hyaluronic acid, $\alpha_{2}$ macroglobulin, age and sex produced areas under the ROC curves of $0.85,0.96$ and 0.94 for significant fibrosis, advanced fibrosis, and cirrhosis, respectively.In the training set, the model was $\mathbf{9 2 \%}$ specific and $67 \%$ sensitive for significant fibrosis, $\mathbf{8 1 \%}$ specific and $\mathbf{9 5 \%}$ sensitive for advanced fibrosis, and $84 \%$ specific and $\mathbf{7 1 \%}$ sensitive for cirrhosis. Thus it provides clinically useful information regarding different fibrosis stages among hepatitis $\mathrm{C}$ patients [66]

5- SHASTA Index: The SHASTA index consists of serum hyaluronic acid, AST, and albumin.It was evaluated in patients with HIV/HCV coinfection.As with other biomarker assays, optimal results were noted in the extreme categories. The SHASTA index in HIV/HCV has similar accuracy to the Fibrotest and this test is significantly better than the APRI test [67]

6- APRICOT Clinical Investigators Assay: A retrospective analysis of liver biopsies was performed in $\mathbf{8 3 2}$ patients with $\mathrm{HIV} / \mathrm{HCV}$ co-infection, who were randomly assigned to training $(\mathrm{n}=\mathbf{5 5 5})$, and validation $(\mathrm{n}=\mathbf{2 7 7})$ sets. The authors derived a simple index (FIB-4):Age ([yr] $\times$ AST $[\mathrm{U} / \mathrm{L}]) /\left(\left(\mathrm{Plt}\left[\mathbf{1 0}^{\mathbf{9}} / \mathrm{L}\right]\right) \times(\mathrm{ALT}\right.$ $[\mathrm{U} / \mathrm{L}]) \times(\mathbf{1} / \mathbf{2}))$

The AUROC of the index was $\mathbf{0 . 7 6 5}$ for differentiation between Ishak stage $\mathbf{0}$ 3 and 4-6 .At a cut off of $<\mathbf{1 . 4 5}$ in the validation set the negative predictive value to exclude advanced fibrosis (stage 4-6)was $90 \%$ with a sensitivity of $70 \%$.A cut off of $>3.25$ had a positive predictive value of $65 \%$ and a specificity of $97 \%$.Using these cutoffs, $87 \%$ of the 198 patients with FIB-4 values outside 1.45-3.25 would be correctly classified, and liver biopsy could be avoided in $\mathbf{7 1} \%$ of the validation group [68].

Assessing Serum Model Performance: Serum models are assessed against the prevailing liver biopsy gold standard, although it is a flawed standard. [57]Assessed the risk factors for discordances between the FibroTest serum marker model and biopsy, and then classified them as attributable to either biopsy or marker failure.Discordance was attributable to failure of serum markers in $\mathbf{2 . 4 \%}$, to biopsy failure in $\mathbf{1 8 \%}$ and was not attributable in a further $\mathbf{8 \%}$ of patients. The most frequent reason for marker failure was a false negative result due to inflammation affecting the serum results, whereas biopsy failures were usually due to false negative staging associated with smaller biopsy size, fragmented biopsy and steatosis.In a similar study, discordance was attributable to failure of FibroTest serum marker model in 5\%, to biopsy failure in $4 \%$ and was not attributable in a further $9 \%$ of patients [69]. 


\section{References}

1. B. J. McMahon, "The natural history of chronic hepatitis B virus infection," Hepatology, 2009:vol. 49, no. 5: S45-S55.

2. D. Lavanchy, "The global burden of hepatitis

C," Liver International,2009; vol. 29; 74-81.

3. A. C. Moorman, S. C. Gordon, L. B. Rupp et al., "Baseline characteristics and mortality among people in care for chronic hepatitis: the chronic hepatitis cohort study," Clinical Infectious Diseases,2012; vol. 56, no. $1 ; 40-50$,

4. F. Cainelli, "Liver diseases in developing countries," World Journal of Hepatology, vol. 4, no. 3; 66-67, 2012.

5. X. Hu, Y. Huang, Z. Bao et al., "Prevalence and factors associated with nonalcoholic fatty liver disease in shanghai work-units," BMC Gastroenterology,2012; vol. 12, article 123.

6. A. A. Bravo, S. G. Sheth, and S. Chopra, "Liver biopsy," The New England Journal of Medicine,2001; vol. 344, no. 7; 495-500,.

7. A. Baranova, P. Lal, A. Birerdinc, and Z. M. Younossi, "Non-invasive markers for hepatic fibrosis," BMC Gastroenterology,

2011;vol. 11; article 91.

8. R. Bataller and D. A. Brenner, "Liver fibrosis," The Journal of Clinical Investigation,2005; vol. 115, no. 2; 209-218.

9. R. G. Knodell, K. G. Ishak, and W. C. Black, "Formulation and application of a numerical scoring system for assessing histological activity in asymptomatic chronic active hepatitis," Hepatology,1981; vol. 1, no. $5 ; 431-435$.
10. R. A. Standish, E. Cholongitas, A. Dhillon, A. K. Burroughs, and A. P. Dhillon, "An appraisal of the histopathological assessment of liver fibrosis," Gut,2006; vol. 55, no. 4;569-578.

11. P. J. Scheuer, "Classification of chronic viral hepatitis: a need for reassessment," Journal of Hepatology,1991; vol. 13, no. 3; 372-374.

12. L. Castera, "Invasive and noninvasive methods for the assessment of fibrosis and disease progression in chronic liver disease," Best Practice and Research: Clinical Gastroenterology,2011; vol. 25, no. 2; 291-303.

13. Myers RP, Fong A, Shaheen AA. Utilization rates, complications and costs of percutaneous liver biopsy: a population-based study including 4275 biopsies. Liver Int. 2008;28:705-712. [PubMed]

14. 2. Terjung $B$, et al. Bleeding complications after percutaneous liver biopsy. An analysis of risk factors. Digestion. 2003;67:138145. [PubMed]

15. 3. Bravo AA, Sheth SG, Chopra S. Liver biopsy. The New England journal of medicine. 2001;344:495500. [PubMed]

16. 4. Regev A, et al. Sampling error and intraobserver variation in liver biopsy in patients with chronic $\mathrm{HCV}$ infection. Am.

$\mathrm{J}$.

Gastroenterol. 2002;97:2614-

2618. [PubMed]

17. 5. Ratziu V, et al. Sampling variability of liver biopsy in nonalcoholic fatty liver disease. Gastroenterology. 2005;128:

1898-1906. [PubMed]

18. 6. Olsson R, et al. Sampling variability of percutaneous liver 
biopsy in primary sclerosing cholangitis. J. Clin. Pathol. 1995;48:933-935. [PMC free article] [PubMed]

19. J. Parkes, I. N. Guha, P. Roderick, and W. Rosenberg, "Performance of serum marker panels for liver fibrosis in chronic hepatitis C," Journal of Hepatology, 2006; vol. 44, no. 3; 462-474.

20. M. Pinzani, F. Vizzutti, U. Arena, and F. Marra, "Technology Insight: noninvasive assessment of liver fibrosis by biochemical scores and elastography," Nature Clinical Practice Gastroenterology \& Hepatology,2008; vol. 5, no. 2; 95106.

21. H. H. Chi, C. Verveer, B. E. Hansen, P. E. Zondervan, H. L. A. Janssen, and R. J. de Knegt, "Exclusion of the percutaneous liver biopsy from the management of chronic hepatitis $\mathrm{B}$ and $\mathrm{C}$ patients: are essential secondary diagnosis being missed?" Journal of Hepatology,2012; vol. 56, supplement 2; S411-S412.

22. Grigorescu M. Non-invasive Biochemical Markers of Liver Fibrosis. J Gastrointestine and Liver Dis June 2006; 15: 149-159.

23. Afdhal NH. Diagnosing fibrosis in hepatitis $\mathrm{C}$ : is the pendulum swinging from biopsy to blood tests? Hepatology 2003;37:972-974.

24. Alberti A, Benvegnu L, Boccato S, et al., Natural history of initially mild chronic hepatitis C. Dig Liver Dis 2004;36:646-654.

25. Strader DB, Wright T, Thomas DL et al., Diagnosis, management, and treatment of hepatitis C. Hepatology 2004; 39:1147-1171.

26. Fried MW, Shiffman ML, Reddy KR et al. Peginterferonalfa 2-b plus ribavirin for chronic hepatitis $\mathrm{C}$ virus infection. N Eng J Med 2002; 347:975-982.

27. Afdhal, NH. andNunes, D. Evaluation of liver fibrosis: a concise review. Am J Gastro- enterol; 2004. 99(6):1160-1174.

28. McCourt PA, Smedsrod BH, Melkko $\mathrm{J}$, et al. Characterization of a hyaluronan receptor on rat sinusoidal liver endothelial cells and its functional relationship to scavenger receptors. Hepatology 1999;30: 127686.

29. Idobe $Y$, Murawaki Y, Ikuta $Y$, et al. Post-prandial serum hyaluronan concentration in patients with chronic liver disease. Intern Med 1998; 37:568-75.

30. Schuppan D, Stölzel U, Oesterling C, et al., Serum assay for liver fibrosis. J Hepatol 1995; 22: 82-88.

31. Fried MW, Shiffman ML, Reddy KR et al. Peginterferonalfa 2-b plus ribavirin for chronic hepatitis $\mathrm{C}$ virus infection. N Eng J Med 2002; 347:975-982.

32. Murawaki Y, Ikuta $Y$, Nishimura $Y$ et al. Serum markers for connective tissue turnover in patients with chronic hepatitis $\mathrm{B}$ and chronic hepatitis $\mathrm{C}$ : a comparative analysis. $\mathrm{J}$ Hepatol 1995; 23: 145-152.

33. Eriksson S, Fraser JR, Laurent TC, et al.endothelial cells are asite of uptake and degradation of hyaluronic acid in the liver. Experimental Cell Research 1983; 144: 223-228.

34. Guechot J, Loria A, Serfaty L, et al. Hyaluronan as a marker of liver fibrosis in chronic viral hepatitis $\mathrm{C}$ : effect of alpha-interferon therapy. J Hepatol 1995; 22:22-26.

35. Johansen JS, Christoffersen $P$, Moller S et al. Serum YKL-40 is increased in patients with hepatic 
fibrosis. J Hepatol 2000; 32:911-920.

36. Tran A, Benzaken S, Saint-Paul MC, et al. Chondrex (YKL-40), a potential new serum fibrosis marker in patients with alcoholic liver disease. Eur J GastroenterolHepatol2000 ; ;2:98993.

37. Arthur MJP. Fibrosis and matrix degradation. Digestion 1998; 59: 376380 .

38. Friedman SL. Evaluation of fibrosis and hepatitis C. Am J Med 1999; 107:S27-S30

39. Benyon D and Arthur MJ. Extracellular matrix degradation and the role of stellate cells. Semin. Liver Dis2001; 21: 373-384.

40. Zhang BB, Min CAI W, Weng $\mathrm{HL}$ et al. Diagnostic value of platelet derived growth factor-BB, transforming growth factor-â1, matrix metalloproteinase- 1 , and tissue inhibitor of metalloproteinase-1 in serum and peripheral blood mononuclear cells for hepatic fibrosis. World J Gastroenterol 2003; 2490-2496

41. Sasaki H, Pollard RB, Schmitt D, et al., Transforming growth factor $-\beta$ in the regulation of immune response. ClinImmunolImmunopathol 1992; 65:1-9.

42. Breitkopf $\mathrm{K}$, Lahme B, Tag CG and Gressner AM. Expression and matrix deposition of latent transforming growth factor beta binging proteins in normal and fibrotic rat liver and transdifferentiating hepatic stellate cells in culture. Hepatology 2001; 33: 387-396.

43. Nellson DR, Gonzales-Peralta RP, Qian $\mathrm{K}$ et al .Transforming growth factor- $\beta 1$ in chronic hepatitis . J Viral Hepat 1997; 4: 29-35.
44. Kanzler S, Beumann M, Schirmacher $\mathrm{P}$ et al. Prediction of progressive liver fibrosis in hepatitis $\mathrm{C}$ infection by serum and tissue levels of transforming growth factor $\beta$. J Viral Hepat 2001; 8: 430-438.

45. Pinzani $\mathrm{M}$ and Marra F. Cytokine receptors and signaling in hepatic stellate cells. Semin Liver Dis 2001; 21: 397-416.

46. Kaplanski G, Farnarier C, Payan $\mathrm{MJ}$, et al. Increased levels of soluble adhesion molecules in the serum of patients with hepatitis $\mathrm{C}$ : Correlation with cytokine concentrations and liver inflammation and fibrosis. Dig Dis Sci 1997; 42: 2277-84.

47. Naveau S, Poynard T, Benattar C et al. Alpha-2-macroglobulin and hepatic fibrosis. Diagnostic interest Dig Dis Sci 1994;39:2426-32

48. Poynard T, Aubert A, Bedossa P, et al.,A simple biological index for detection of alcoholic, liver disease in drinkers. Gastroenterology 1991;100:1397-1402.

49. Imbert-Bismut $\mathrm{F}$, Ratziu $\mathrm{V}$, Pieroni $\mathrm{L}$ et al. Biochemical markers of liver fibrosis in patients with hepatitis $\mathrm{C}$ virus infection: a prospective study. Lancet 2001;357:1069-1075.

50. Grigorescu M. Non-invasive Biochemical Markers of Liver Fibrosis. J Gastrointestine and Liver Dis June 2006; 15: 149-159.

51. Pradat $P$, Alberti A, Poynard $T$ et al. Predictive value of ALT levels for histologic findings in chronic hepatitis C: a European Collaborative Study. Hepatology 2002; 36:973-977.

52. Okuda M, Li K, Beard MR, et al. Mitochondrial injury, oxidative stress and antioxidant gene expression are induced by hepatitis $\mathrm{C}$ virus core protein. Gastroenterology 2002; 122: 
366-75.

53. Gordon SC, Fang J W, Silverman AL. et al. The significance of baseline serum alanine aminotransferase on pretreatment disease characteristics and response to therapy in chronic hepatitis C. Hepatology 2000; 32: 400-404.

54. Giannini E, Risso D, Botta $F$ et al. Validity and clinical utility of the aspartate aminotransferase-alanine aminotransferase ratio in assessing disease severity and prognosis in patients with hepatitis $\mathrm{C}$ virus-related chronic liver disease. Arch Intern Med 2003; 163: 218-224.

55. Peck-Radoslavljevic Hypersplenism. Eur M. GastroenterolHepatol 2001; 13: 317 323.

56. Pilette $\mathrm{C}$, Oberti $\mathrm{F}$, Aube $\mathrm{C}$ et al. Non invasive diagnosis of esophageal varices in chronic liver disease. J Hepatol 1999; 31: 867-873.

57. Oberti F, Valsesia E, Pilette C, et al. Non-invasive diagnosis of hepatic fibrosis or cirrhosis. Gastroenterology 1997; 113: 1609-16.

58. Kelleher TB and Afdhal N. Noninvasive Assessment of Liver Fibrosis. Clinics in Liver Disease 2005; 9: 667-683.

59. Poynard $\mathrm{T}, \mathrm{McHutchison} \mathrm{J}$, Manns M, et al. Biochemical surrogate markers of liver fibrosis and activity in a randomized trial of peginterferonalfa 2-b and ribavirin. Hepatology 2003; 38: 481-92.

60. Poynard T, Ratziu V, Naveau S, et al. The diagnostic value of biomarkers(steatoTest) for the prediction of liver steatosis. Comp Hepatol 2005; 4:10.

61. Forns X, Ampurdanès S., Llovet JM., et al., Identification of chronic hepatitis C patients without hepatic fibrosis by a simple predictive model. Hepatology, 2002;36:986-992.

62. Wai CT, Greenson JK, Fontana $\mathrm{RJ}$, et al. A simple non-invasive index can predict both significant fibrosis and cirrhosis in patients with chronic hepatitis C. Hepatology 2003; 38: 518-26.

63. Islam S, Antonsson L, Westin J, et al. Cirrhosis in hepatitis $\mathrm{C}$ virus infected patients can be excluded using an index of standard biochemical serum markers. Scand J Gastroenterol 2005; 40: 867-872.

64. Patel K, Gordon SC, Jacobson I, et al. Evaluation of a panel of noninvasive serum markers to differentiate mild from moderate-toadvanced liver fibrosis in chronic hepatitis C patients. J Hepatol 2004;41:935-942.

65. Mehta P, Ploutz-Snyder R, Nandi J, et al. Diagnostic accuracy of Serum Hyaluronic Acid, FIBROSpect II, and YKL-40 for discriminating fibrosis stages in chronic hepatitis C. Am J Gastroenterol 2008; 103: 928-36.

66. Rosenberg WM, Voelker $\mathrm{M}$, Thiel R, et al. Serum markers detect the presence of liver fibrosis: a cohort study. Gastroenterology 2004; 127:1704-13.

67. Calès $\mathrm{P}$, Oberti $\mathrm{F}$, Michalak $\mathrm{S}$, et al. A novel panel of blood markers to assess the degree of liver fibrosis. Hepatology 2005; 42: 1373-1381.

68. Adams LA, Bulsara M, Rossi E. Hepascore: an accurate validated predictor of liver fibrosis in chronic hepatitis C infection. ClinChem 2005; 51: 1867-1873.

69. Kelleher TB and Afdhal N. Noninvasive Assessment of Liver Fibrosis. Clinics in Liver Disease 2005; 9: 667-683.

70. Sterling RK, Lissen E, Clumeck 
$\mathrm{N}$, et al., Development of a simple noninvasive index to predict significant fibrosis in patients with HIV/HCV coinfection. Hepatology, 2006; 43:1317-1325.

71. Halfon P, Bourliere M, Deydier $\mathrm{R}$, et al. Independent prospective multicenter validation of biochemical markers (fibrotest-actitest) for the prediction of liver fibrosis and activity in patients with chronic hepatitis C: the fibropaca study. Am J Gastroenterol 2006; 101:547-55. 